\title{
New-Onset Uveitis Possibly Caused by Secukinumab in a 47-Year-Old Male Patient with Long-Standing Ankylosing Spondylitis
}

This article was published in the following Dove Press journal: International Medical Case Reports Journal

\author{
Huda Nadwi (D) \\ Murad Janaini (iD) \\ Mohammed Zammo (iD ${ }^{2}$ \\ Mohamed Cheikh iD ${ }^{2}$ \\ Hani Almoallim (D) ${ }^{1-3}$ \\ 'Department of Medicine, College of \\ Medicine, Umm Alqura University, \\ Makkah, Saudi Arabia; ${ }^{2}$ Department of \\ Medicine, Dr. Soliman Fakeeh Hospital, \\ Jeddah, Saudi Arabia; ${ }^{3}$ Alzaidi Chair of \\ Research in Rheumatic Diseases, Umm \\ Alqura University, Makkah, Saudi Arabia
}

Correspondence: Hani Almoallim Department of Medicine, College of Medicine, Umm Alqura University, P.O. Box 182I, Jeddah 2144I, Saudi Arabia Tel +966505703935

Email hmmoallim@uqu.edu.sa

\begin{abstract}
Secukinumab, "an IL-17 antagonist", is one of the biological agents used to treat active ankylosing spondylitis (AS). Although it has been proven that certain agents are linked with a paradoxical increase in uveitis, there are limited data on whether secukinumab has this effect or not. We report a case of a new-onset anterior uveitis after 6 months of starting secukinumab in a 47-year-old male, HLA-B27 positive AS patient. He had a long-standing history with the disease over 25 years. He was treated in the past with methotrexate then adalimumab and later on with etanercept. He had no history of uveitis during all of this time. The uveitis was mild and treated conventionally with local measures while secukinumab was maintained. After a close follow-up, the uveitis had completely resolved. Is this part of the original disease or a possible side effect from secukinumab?
\end{abstract}

Keywords: spondyloarthritis, iritis, IL-17A inhibitors, biologics, ankylosing spondylitis, uveitis, secukinumab

\section{Introduction}

Ankylosing spondylitis (AS) is a chronic inflammatory autoimmune disease affecting the axial skeleton, particularly the sacroiliac joints. ${ }^{1}$ Some of its most characteristic symptoms are stiffness, chronic back pain, and loss of spinal mobility. If left untreated, it can cause total fusion of the axial skeleton, leading to disability and impaired quality of life. AS is known to have many extra-articular manifestations, the most common of which is anterior uveitis. ${ }^{2}$

Unless the flares are numerous, most patients with acute anterior uveitis are treated with topical steroid therapy and retain good visual acuity. It is a serious manifestation, and if kept untreated, it can lead to multiple complications that threaten the sight and can even lead to blindness.

Secukinumab, a monoclonal antibody, is an IL-17A inhibitor, the first of its kind to be approved for the treatment of AS. So far it has been shown to be generally effective regardless of previous TNF inhibitor use. The ASAS-European League Against Rheumatism (EULAR) guidelines that were recently updated (in 2015) now recommend the use of biologic DMARD's including IL-17 inhibitor in patients with whom conventional treatment has failed. ${ }^{3}$

While biologic therapies have been employed in addition to conventional therapy for uveitis, the effectiveness of secukinumab in particular for the treatment of uveitis has not been established. ${ }^{4}$ Here, we report a case of a new-onset uveitis 
after starting secukinumab in a patient with a longstanding AS, who had no previous extra-articular manifestations. Institutional review board was not required to publish this case report. The patient's written informed consent was obtained to publish this case report.

\section{Case Report}

A 47-year-old male patient, diagnosed with AS 25 years ago with a main presenting complaint of gradually progressive neck and back pain. This was associated with morning stiffness, lasting more than 1 hour, affecting his daily activity with limited range of motion and improving on nonsteroidal anti-inflammatory drugs (NSAIDs). He had no other joints' involvement, ocular pain, redness or any acute visual disturbance, no shortness of breath, chest pain, abdominal pain, or bowel disturbances. There was no skin rash or lesions. He was not known to have any medical illnesses before. He sought multiple medical advice and was diagnosed with AS based on bilateral sacroiliitis on MRI. He is HLA-B27 positive.

Initially, he was started on methotrexate by a rheumatologist for 1 year hoping that it might help his back pain. There was no significant improvement in his symptoms. Another rheumatologist shifted him to adalimumab $40 \mathrm{mg}$ per 2 weeks due to the persistence of pain and lack of efficacy of methotrexate. He showed significant improvement with adalimumab. Later on, after 2 years of treatment, adalimumab was discontinued, and he was started on etoricoxib $60 \mathrm{mg}$ once daily alternating with celecoxib $200 \mathrm{mg}$ twice daily and topical diclofenac with minimal improvement. Adalimumab was resumed by his rheumatologist after 6 months due to an active disease, patient had considerable improvement and he remained on this regimen with avid exercise with no mentionable changes of complications. He had a flare of his disease in 2011 despite being on adalimumab and he was switched to etanercept $50 \mathrm{mg}$ per week, during this time he suffered from recurrent infections in the form of skin abscesses and recurrent sinusitis. There were episodes of holding the treatment until his infections were cured and then resuming etanercept. His recurrent infections were bothering, and subsequently, he was started on secukinumab $150 \mathrm{mg}$ per week for a total of five loading doses and he did not notice a major improvement in his symptoms then it was increased to $300 \mathrm{mg}$ once monthly in May 2019. Later on, in Nov 2019 and after a total of 11 doses, he presented to an ophthalmologist with cloudy vision and painful red eye. He was diagnosed with the first episode of anterior uveitis. He denied any previous similar complaints or symptoms and after excluding all other infectious causes such as TB, syphilis, and HSV he was started on local corticosteroid eye drops for 2 months. The patient was reluctant to initiate systemic corticosteroid and he was maintained on secukinumab $150 \mathrm{mg}$ once monthly with close monitoring in the clinics. His eye symptoms started to improve by the first week of local treatment and by the fifth week, his uveitis was totally resolved. His disease remained controlled while he was maintained on secukinumab. He showed no major limitations in movement. His C-reactive protein (CRP) was normal. His disease was mainly monitored based on symptoms and other objective measures such as the Bath Ankylosing Spondylitis Disease Activity Index (BASDAI). His BASDAI score was 0.9. Physical examination in his last visit in Feb 2020 showed limitation of his lumbar spine flexion with a positive modified Schober test. He also demonstrated mild limitation in lateral flexion, chest expansion, and occiput to wall distance of $5 \mathrm{~cm}$. His medication was maintained even during the era of COVID-19 pandemic and he had no more ocular symptoms.

\section{Discussion}

We report here a case of a new-onset uveitis after 6 months of using secukinumab in a patient with a long-standing AS, who had no previous history of similar attacks. Uveitis is the most common extra-articular manifestations of AS with a prevalence of $25.8 \%$ based on one metaanalysis, ${ }^{5}$ even reported to be reaching $30-40 .{ }^{6}$ It is commonly known that the first episode of uveitis occurs between the age of $20-40 .^{7}$ While some studies have shown that the occurrence of uveitis is associated with longer disease duration like in our patient reported here, other studies failed to support this association. ${ }^{8}$

Our patient had never experienced any episode of uveitis throughout 25-year history of AS. He had used adalimumab and etanercept intermittently during this time. He developed his first episode of uveitis only after 6 months of using secukinumab. This brings to our attention the only new factor in the equation, which is the recent use of secukinumab. The establishment of the first episode of uveitis within just 6 months of starting secukinumab raised the question of whether secukinumab was the underlying cause of uveitis, or it was simply the first extra-articular manifestation of AS?

Secukinumab is generally a well-tolerated treatment option; nevertheless, like any other treatment, it has adverse effects including increased risk of infection. In a 5-year extension trial across multiple domains of AS, 
efficacy was sustained and the safety profile was consistent. ${ }^{12}$ There is no widely available data documenting secukinumab as the underlying cause of uveitis. There is, however, one reported case of a paradoxical flare of psoriatic uveitis after switching from infliximab to secukinumab. ${ }^{9}$ This sheds light on the possibility that anti- IL17 drugs may also be associated with paradoxical worsening of disease including uveitis. The incidence rate of uveitis (new-onset and flares) is low 1.4 patient-year in a pooled analysis of 3 randomized control trials for secukinumab. ${ }^{13}$ Apparently reports from real-world data are still lacking in this matter. We report here one of the real-world experiences with the use of secukinumab.

Since the introduction of etanercept (TNF receptor-Fc fusion protein) in 1998, it has proven to be an effective treatment for AS, The British Society of Rheumatology (BSR) guidelines of 2010 allowed its use even in patients with uveitis. However, the growing number of reports implicating it as the cause of new-onset uveitis or a flare of existing uveitis eventually established that etanercept may cause uveitis as a paradoxical effect. This revelation resulted in the 2019 American College of Rheumatology (ACR) guidelines that recommend switching to monoclonal antibodies in case of a uveitis flare instead of etanercept. ${ }^{4}$ Given this experience, we believe the possibility of secukinumab also causing uveitis as a paradoxical effect is something to consider. It may be too early to conclude at this point, but we are waiting for more experience to be published with secukinumab.

Although the current recommendations regarding the mode of administration of secukinumab in AS are subcutaneous (SC) doses, a study comparing intravenous (IV) and SC doses shows that IV is much more effective in the treatment of noninfectious uveitis, and that SC doses may be insufficient. ${ }^{10}$ It has to be noted that the cases of uveitis included in this study were not homogenous but variable with different etiologies. Assuming this episode of uveitis reported in our patient here was indeed an extra-articular manifestation, perhaps it was undertreated by an insufficient dose of SC secukinumab, and could have been prevented had he been treated with IV instead?

A recent review of three randomized controlled clinical trials studying the effectiveness of secukinumab shows that while it may help reduce the use of immunosuppressive medications, in terms of recurrence of uveitis, it showed no significant difference between treatment and placebo groups. ${ }^{11}$ This review has been criticized for involving an inhomogeneous group of populations with different types of uveitis and different underlying conditions including those with Behçet's disease and those with other diseases. It should be noted that subcutaneous secukinumab was used in all three of these trials. Based on the former study mentioned, this once again begs the question, would there have been a reduction in the rate of recurrence had they used IV instead? This needs to be validated in well-designed randomized controlled trials to assess the optimum mode of administration of secukinumab in patients with AS.

In such cases of uncertainty, where the cause of uveitis is not clear, it is usually treated by discontinuing the offending drug, starting local or systemic steroids depending on the severity and initiating a different mode of therapy with immunosuppressive medications such as sulfasalazine or methotrexate. The treatment plan for our patient was the result of a great collaboration between the treating ophthalmologist and rheumatologist. It was decided that there was no need for systemic steroids as it was a mild flare. He was treated then with topical steroids while maintaining the minimum effective dose of secukinumab with close follow-up visits to determine the response to treatment. The patient was followed in the clinic and successfully established a complete resolution of his active acute uveitis. The patient had been maintained on secukinumab over the period of COVID-19 with no new flares.

Uveitis can have serious consequences. Physicians should be aware of this extra-articular manifestation of AS and its many etiologies. One such etiology to be considered is the recently discovered paradoxical effect of some biological medications used to treat AS. Secukinumab may potentially have a similar effect. It is not clear whether secukinumab is the culprit behind our patient's flare; still, we did not elect to discontinue it despite the mild episode of uveitis. The paradoxical effect of secukinumab is an area for further research to be conducted.

\section{Funding}

This work was funded and supported by Alzaidi Chair of Research in Rheumatic Diseases, Umm Alqura University, Makkah, Saudi Arabia.

\section{Disclosure}

Hani Almoallim reports this work was supervised and funded by Alzaidi Chair of Research in Rheumatic, Umm Alqura University, Makkah, Saudi Arabia. The 
authors report no other potential conflicts of interest for this work.

\section{References}

1. Braun J, Sieper J. Ankylosing spondylitis. Lancet. 2007;369 (9570):1379-1390. doi:10.1016/S0140-6736(07)60635-7

2. El Maghraoui A. Extra-articular manifestations of ankylosing spondylitis: prevalence, characteristics and therapeutic implications. Eur J Intern Med. 2011;22(6):554-560. doi:10.1016/j.ejim.2011.06.006

3. Ward MM, Deodhar A, Akl EA, et al. American College of Rheumatology/Spondylitis Association of America/Spondyloarthritis Research and Treatment Network 2015 Recommendations for the Treatment of Ankylosing Spondylitis and Nonradiographic Axial Spondyloarthritis. Arthritis Rheumatol. 2016;68(2):282-298. doi: $10.1002 /$ art.39298

4. Ward MM, Deodhar A, Gensler LS, et al. 2019 Update of the American College of Rheumatology/Spondylitis Association of America/Spondyloarthritis Research and Treatment Network Recommendations for the Treatment of Ankylosing Spondylitis and Nonradiographic Axial Spondyloarthritis. Arthritis Care Res. 2019;71 (10):1285-1299. doi:10.1002/acr.24025

5. Stolwijk C, van Tubergen A, Castillo-Ortiz JD, Boonen A. Prevalence of extra-articular manifestations in patients with ankylosing spondylitis: a systematic review and meta-analysis. Ann Rheum Dis. 2015;74 (1):65-73. doi:10.1136/annrheumdis-2013-203582

6. Rosenbaum JT. Uveitis in spondyloarthritis including psoriatic arthritis, ankylosing spondylitis, and inflammatory bowel disease. Clin Rheumatol. 2015;34(6):999-1002. doi:10.1007/s10067-015-2960-8
7. Chang JH, McCluskey PJ, Wakefield D. Acute anterior uveitis and HLA-B27. Surv Ophthalmol. 2005;50(4):364-388. doi:10.1016/j. survophthal.2005.04.003

8. Wendling D, Joshi A, Reilly P, Jalundhwala YJ, Mittal M, Bao Y. Comparing the risk of developing uveitis in patients initiating anti-tumor necrosis factor therapy for ankylosing spondylitis: an analysis of a large US claims database. Curr Med Res Opin. 2014;30(12):2515-2521. doi:10.1185/03007995.2014.969368

9. Su P, Pan JY. Paradoxical flare of psoriasis, psoriatic spondyloarthritis, and psoriatic uveitis after switching from infliximab to secukinumab. Dermatol Sin. 2017;35(2):112-113. doi:10.1016/j. dsi.2016.10.002

10. Letko E, Yeh S, Foster CS, Pleyer U, Brigell M, Grosskreutz CL. Efficacy and safety of intravenous secukinumab in noninfectious uveitis requiring steroid-sparing immunosuppressive therapy. Ophthalmology. 2015;122(5):939-948. doi:10.1016/j.ophtha.2014.12.033

11. Dick AD, Tugal-Tutkun I, Foster S, et al. Secukinumab in the treatment of noninfectious uveitis: results of three randomized, controlled clinical trials. Ophthalmology. 2013;120(4):777-787. doi:10.1016/j. ophtha.2012.09.040

12. Baraliakos X, Braun J, Deodhar A, et al.; on behalf of the MEASURE 1 study group. Long-term efficacy and safety of secukinumab $150 \mathrm{mg}$ in ankylosing spondylitis: 5-year results from the Phase III MEASURE 1 extension study. RMD Open. 2019;5: e001005. doi:10.1136/rmdopen-2019-001005

13. Deodhar AA, Miceli-Richard C, Baraliakos X, et al. Incidence of uveitis in secukinumab-treated patients with ankylosing spondylitis: pooled data analysis from three Phase 3 studies. ACR Open Rheumatol. 2020;2(5):294-299. doi:10.1002/acr2.11139

\section{Publish your work in this journal}

The International Medical Case Reports Journal is an international, peer-reviewed open-access journal publishing original case reports from all medical specialties. Previously unpublished medical posters are also accepted relating to any area of clinical or preclinical science. Submissions should not normally exceed 2,000 words or 4 published pages including figures, diagrams and references. The manuscript management system is completely online and includes a very quick and fair peer-review system, which is all easy to use. Visit http://www.dovepress.com/testimonials.php to read real quotes from published authors. 